\title{
Erweiterung des Treuhandpartnernetzes im Kanton Zürich
}

Die eisele \& partner treuhand ag, die neue Vertrauenspartnerin FMH Services (Treuhand) mit Sitz in Winterthur, ist bereits seit 1990 eine fachlich versierte Ansprechpartnerin in allen Fragen des Treuhandwesens und der Wirtschaftsprüfung. Ihr kompetentes Team umfasst 14 Mitarbeiterinnen und Mitarbeiter vom Sachbearbeiter bis hin zum eidg. dipl. Wirtschaftsprüfer und Experten in Rechnungswesen und Controlling. Die eisele \& partner treuhand ag ist Mitglied im schweizerischen Treuhänderverband (TREUHAND SUISSE) sowie im Expertenverband für Wirtschaftsprüfung, Steuern und Treuhand (EXPERTsuisse). Dadurch verpflichtet sie sich zur Einhaltung deren Standes- und Berufsregeln, was unter anderem eine hohe Sorgfalt, Verschwiegenheit und Unabhängigkeit garantiert.

Das Dienstleistungsangebot der eisele \& partner treuhand ag umfasst sämtliche Bereiche des Treuhandwesens und der Wirtschaftsprüfung. Neben dem Führen der Finanz- und Betriebsbuchhaltung, welche bei Bedarf durch eine Debitoren- und/oder Kreditorenbuchhaltung inklusive Zahlungsverkehr ergänzt werden kann, übernehmen Michael Luther und sein Team auch gerne Ihre gesamte Personaladministration und Lohnbuchhaltung. Des Weiteren wird das Angebot durch auf Ihre Bedürfnisse zugeschnittene Budgetund Planrechnungen, Kalkulationen und Analysen ergänzt.

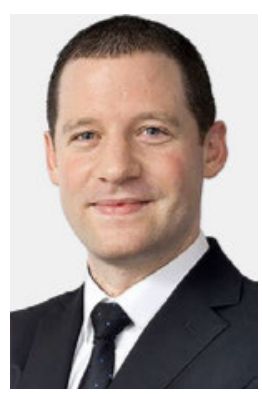

Michael Luther

Dipl. Wirtschaftsprüfer

Treuhänder mit eidg. FA

michael.luther@fmhtreuhand.ch

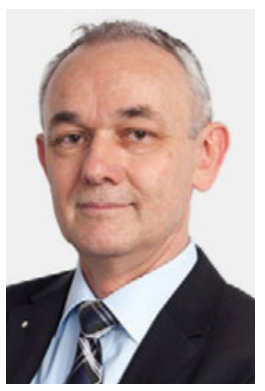

Peter Eisele

Dipl. Wirtschaftsprüfer

Experte für Rechnungswesen

und Controlling

peter.eisele@fmhtreuhand.ch
Im Bereich der Steuern bietet die eisele \& partner treuhand ag massgeschneiderte Beratungen für Sie und Ihr Unternehmen an. Neben dem Ausfüllen der Steuererklärung legt sie grossen Wert auf eine vorausschauende und nachhaltige Planung sämtlicher steuerrelevanten Aspekte. Ebenfalls unterstützt und berät Sie die eisele \& partner treuhand ag gerne bei der Abrechnung der Mehrwertsteuer, Quellensteuer und der Grundstückgewinnsteuer.

Die eisele \& partner treuhand ag ist bei der Revisionsaufsichtsbehörde als zugelassene Revisionsexpertin registriert. Zum Fachgebiet von Michael Luther gehören sowohl ordentliche und eingeschränkte Revisionen als auch interne Prüfungen. Bei Letzterem werden die Effizienz Ihrer Abläufe und Geschäftsprozesse untersucht und Vorschläge zur Steigerung ausgearbeitet. Ebenfalls können Spezialprüfungen, zum Beispiel gemäss Fusionsgesetz, für Sie durchgeführt werden.

Die eisele \& partner treuhand ag legt grössten Wert auf eine persönliche Beratung und auf ein speziell nach Ihren Bedürfnissen zugeschnittenes Angebot. Dank langjähriger Erfahrung werden Sie in sämtlichen administrativen Belangen optimal unterstützt und können sich vollends auf Ihr Kerngeschäft konzentrieren.

Herr Michael Luther und sein Team freuen sich sehr, Vertrauenspartner FMH Services (Treuhand) zu sein und Sie zukünftig betreuen zu dürfen.

eisele \& partner treuhand ag

FMH Services (Treuhand)

Im Hölderli 26

8405 Winterthur

Tel. 05224511 88, Fax 0522451189

eisele-treuhand.ch
Die eisele \& partner treuhand ag ist ein von der FMH Services Genossenschaft empfohlenes, rechtlich und wirtschaftlich selbständiges Beratungsunternehmen. 